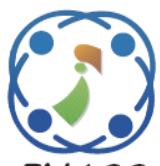

\title{
Solving Capacitated Vehicle Routing Problem Using Chicken Swarm Optimization with Genetic Algorithm
}

\author{
Nora Niazy ${ }^{1,2 *}$ \\ Ahmed El-Sawy ${ }^{1}$ \\ Mahmoud Gadallah ${ }^{2}$ \\ ${ }^{I}$ Computer Science Department, Faculty of Computers and Artificial Intelligence, Benha University, Egypt \\ ${ }^{2}$ Modern Academy for Computer Science and Management Technology, Egypt \\ * Corresponding author's Email: nora_niazy@fci.bu.edu.eg
}

\begin{abstract}
The Capacitated Vehicle Routing Problem is the most popular type of Vehicle Routing Problem and is a kind of NP-hard problems. Finding the minimum total distance travelled by vehicles to serve a group of customers with respect to capacity constrains is the aim of the Capacitated Vehicle Routing Problem. This problem will be solved by hybrid algorithm combining Chicken Swarm Optimization algorithm with Genetic Algorithm using Crossover and Mutation operation. The main idea of the proposed algorithm is to use the hieratical order of Chicken Swarm Algorithm to find paths after using the moving equations. Then we will rearrange the hieratical order according the paths cost. In an attempt to improve results for some chickens, we will use the Genetic Algorithm because it has the advantage that it searches in the neighbourhood to find the best solution then we will get the best solution which has the lowest cost. Results from a computational experiment on 10 different datasets show that the hybrid algorithm can be considered as an efficient approach and overcome the best known results in 10 datasets which means that it is $100 \%$ better than best known results which exist on NEO benchmark.
\end{abstract}

Keywords: Capacitated vehicle routing problem, Chicken swarm optimization, Genetic algorithm, Particle swarm optimization, Mutation, Crossover.

\section{Introduction}

One of the most important challenges facing people today is the problem of transportation. Many organizations spend a lot of money trying to reduce the cost resulting from using vehicles to deliver goods to their customers. The Vehicle Routing Problem (VRP) can be considered as one of the popular kind of transportation problems. Although the vehicle routing problem has been studied for more than 70 years, the challenge of solving it has increased as more difficult variants arise in the prominent area of development [1]. The VRP is used to find and discover the shortest path for vehicles that serve a group of customers according to some conditions that must be taken into account while performing the service. This type of problem is considered as difficult and very complex problem (NP-hard Problem) that has attracted many scientists and researchers to find multiple ways to solve it $[1,2]$. In
Fig. 1, we can see a simplified form of the VRP in which a path is drawn for a vehicle or a group of vehicles, this path is characterized as the shortest path that can be taken with the service of all customers, noting that the path starts and ends at the same distribution point. The problems of the VRP divided into many forms according to the restrictions placed on the problem. These restrictions may relate to time, customers, type of goods transported, etc.

One of the most important types of VRP is Capacitated VRP (CVRP), in this type, pathways to customer service are specified and each path contains a group of customers who are serviced by a specific vehicle. It must be taken into account that each customer service is done once through the specific vehicle. Also, it must be taken into account that the customer requirements do not exceed the storage space of the vehicle. The second type that we have is VRP with Time Window (VRPTW), in this type we must consider the timing assigned to each customer. 


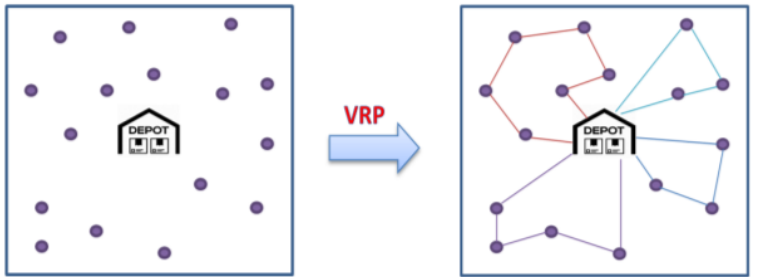

Figure. 1 The VRP is finding a shortest path for vehicles to serve some customers

One of the famous types is VRP with Pickup and Delivery (VRPPD), this type is characterized by accepting the possibility for the customer to return the goods. With this type, the capacity of each vehicle must be able to accommodate the requirements and returns of customers. The last type we will mention is Distance Constraint VRP (DCVRP), this type defines a specific distance for each vehicle so that the specified paths do not exceed the maximum specified distance for the vehicles [3]. In this paper we will solve the CVRP by using hybrid algorithm between Chicken Swarm Optimization (CSO), Tabu Search (TS) and Genetic Algorithm (GA) which give us the advantage of CSO in the chickens' various movements can be led to achieve a good balance between randomness and determinism for finding the optimum, the second advantages are that the whole chicken swarm consists of several groups, namely multi-swarm. Through integration of the hierarchal order, chickens of the different groups may behave as a team and coordinate themselves to forage for food. Thus CSO can behave intelligently to optimize problems efficiently [4]. Also we use the power of TS to avoided local optima where all the neighboring solutions are non-improving [5], also we will use the advantages of GA in applying an iterative and stochastic process on a group of individuals (population), where each individual represents a potential solution to the problem [3]. By using previous advantages for CSO, TS and GA we will get good results that surpass the current best known results. In this work we will propose a hybrid algorithm CSO with Tabu Search and Genetic Algorithm (Crossover and Mutation operations both or one of them). We will mention the basic constraints for CVRP in next section then in section 3 we will talk about the history of the problem as well as the algorithms that we will use to solve the problem.in section 4 we describe how we apply the proposed hybrid algorithm to solve the problem. The experimental results and the comparing between our hybrid algorithm with CSO, Particle Swarm Optimization PSO, and best known results and which algorithm is better will be mentioned is sections 5 .

\section{Problem formulation}

We can consider the problem of VRP is a combinatorial problem and it can be represented as complete directed graph $G(V, E)$, where $V=\left\{V_{d}, V_{c}\right\}$ is a group of vertices including the clients $\left(V_{c}\right)$ and the depots or warehouses $\left(V_{d}\right)$ and $E$ for the connection lines between the customers [6]. Despite the many types of VRP, the problem of CVRP remains the most widespread and studied type of VRP. However, despite the large number of studies that were studied on CVRP, it remains a computationally complex problem and the greater the scale of the problem, the greater the time required to solve it greatly, knowing that the accuracy of the solutions depends on the inputs of the problem in addition to the time given to solve it. We can consider the number of customers, only one central depot, the number of vehicles, the same capacity of these vehicles and the same product are the main components for CVRP. The goal of the CVRP is to put a set of paths that serve all customers through a group of vehicles, and when finding these paths should be as short as possible and also the cost of these paths should be as least as possible, taking into account that each vehicle serves a specific group of customers so that each customer is served once by one vehicle, starting and ending at the same warehouse and the last important thing, the total requirements of customers in each group should not override the storage space for each vehicle [7]. The CVRP can be considered as an undirected graph $G=(V, E)$ where $V=\left\{v 0, v 1, \ldots, v_{n}\right\}$ is a vertex group and $E=\left\{\left(v_{i}, v_{j}\right) / v_{i}, v_{j} \in V, i<j\right\}$ is an edge group. We can consider the depot as Vertex $v o$, and it is from where $y$ correspondent vehicles of capacity should be able to serve every customer, dealing with a group of $n$ vertices $\left\{v l, \ldots, v_{n}\right\}$. We determine on $E$ (a nonnegative cost) distance matrix $X=\left(X_{i j}\right)$ between customers $v_{i}$ and $v_{j}$. Let $V_{l, . .,} V_{m}$ be a split of $V$, a path $P_{i}$ is a permutation of the customers in $V_{i}$ assigning the order of visiting them, starting and finishing at the depot $v_{0}$. The cost of a given path $P_{i}=\left\{v_{i} 0, v_{i l}, \ldots\right.$, $\left.v_{i k+1}\right\}$, where $V I J \in V$ and $v_{i 0}=v_{i k+1}=0$ (0 indicates the depot, $i$ indicates the number of customer ) $[3,8]$. Is given by:

$$
\operatorname{Cost}(P i)=\sum_{j=0}^{k} x_{j, j+1}
$$

And the problem solution $\mathrm{F}(\mathrm{S})$ is:

$$
F_{C V R P}(S)=\sum_{i=1}^{y} \operatorname{Cost}(P i)
$$


The CVRP consists of defining a group of $y$ vehicle paths:

The smallest total cost.

Starting and stopping at the depot $v o$.

Every customer is visited just once by a specific vehicle; according to the restrictions.

Any path doesn't exceed the total requirements:

$$
Q\left(\sum_{v_{j} \in P_{i}} q_{j<Q}\right)
$$

The total distance of any path is not larger than a preset specific

$$
T(\operatorname{Cost}(P i) \leq T)
$$

For all clients the type of product should be the same [3].

The number of vehicles is one of the important elements that effect on the decision taking and the cost of problem. In this work, the path length is reduced separately of the number of used vehicles[9].

\section{Literature review}

We can consider the problem of the VRP a very complex problem and this requires a great time to solve it, and over time a lot of theories have been developed to solve this problem and we will list a set of these solutions that some researchers have come up with. Various methods have been introduced to solve VRP, they can be categorized as exact methods, heuristic algorithms, and metaheuristic algorithms. Exact methods can solve small and medium VRP instances. According to this Constraint, almost algorithms used to solve VRP are heuristic and metaheuristic. Both heuristic and metaheuristic algorithms propose approximate solutions in reasonable computing times, so they are more practical for real-world cases and commercial applications[1]. The next algorithms that we will mention depend on heuristic techniques. a local version PSO based on neighbourhood operator proposed by Suganthan (1999), in the particle swarm optimization algorithm, each particle timely adjusts the position in the searching space according to its flying experience and neighbour node's flying experience [10]. For solving CVRPs Baker. suggest a simple Genetic Algorithm (GA). Bell. introduce Ant Colony Optimization (ACO) algorithm for solving the CVRP. For solving great problems (more than 100 clients) the authors suggest a multiple-ant-colony strategy. An approach which combined two algorithms ACO and Scatter Search was introduced by Zhang. for solving CVRP. In 1995 Eberhart and Kennedy propose a PSO. Their approach is depending on two-stage technique. The first stage is using DPSO to apply the task of clients clustering and the second stage is applying Simulated Annealing (SA) to determine the visiting order for every vehicle [11]. In the particle swarm optimization algorithm, each particle timely adjusts the position in the searching space according to its flying experience and neighbour node's flying experience. The particle swarm is randomly initialized, and the algorithm aims at searching the optimum solution which meets some performance [10]. Robinson, Sinton, and Rahmat-Samii (2002) investigated the possibility of hybridizing PSO and GA to optimize the design of a profiled corrugated horn antenna. They proposed two approaches - GA-PSO and PSO-GA. In GA-PSO approach, GA is applied till improvement in objective function evaluation started to level off and then the GA output is used as the input to PSO [12]. Literature (Xu, Lu, \& Cheng, 2017) Suggested that a sub-path be created and this sub-path contributes to creating the best path and then moving to the second phase of the pheromone application, and use random interpolation method to set the ranking of cities in the optimal solution. It improves the convergence speed and obviate the algorithm falling into local optimal [13]. In the previous works and researches, a single depot with customers distributed around this depot is supposed. The route that was specific for a vehicle, start and end in a centre depot. But sometimes there may be more than one depot and in this case it must be handled by planning various routes covering all the clients or nodes[7, 14]. The majority of the techniques used are heuristic techniques, but the proposed algorithm is a Meta-heuristic algorithm in addition to it is Multi-Swarm Algorithm, which is mean that each sub-swarm focus on specific region while a specific diversification method and this feature gives us a preference in the speed and accuracy of solutions. In a previous work [9], we proposed a hybrid algorithm (CSO with TS) for CVRP, in which more details were mentioned in terms of solving each algorithm for the problem separately, but here we proposed a hybrid CSO,TS and GA in an attempt to obtain much better results than the previous. We will compare the implementation of PSO, CSO and the hybrid CSO with GA algorithms on NEO benchmark datasets. It will become evident to us through the results that the proposed algorithm has exceeded the results of the PSO.

\subsection{Chicken swarm optimization (CSO)}

The CSO was proposed in 2014 by Meng and this algorithm can be considered as Optimization 
algorithm, this algorithm is inspired by the natural life of chickens. The manner of the chicken swarm reckon on hierarchal order. The chicken swarm can be divided into many groups, each group contains one rooster and many hens and chicks. There exist competition between various chickens under certain hierarchical order [4]. Dependence on the hierarchical formation within the swarm is such that this formation is topped by the highest fitness values and in this case this formation is topped by the roosters, and those with the worst fitness values are at the end of the formation and in this case we can consider them the chicks. In the same time, those in the middle are hens. The swarm is divided into groups, each group containing a rooster, a group of hens and a group of chicks and they are generated randomly. The rooster with the highest fitness value can search for food in more places and on a larger scale $[15,16]$.

$$
x_{i, j}^{t+1}=x_{i, j}^{t} \times\left(1+\operatorname{Randn}\left(0, \sigma^{2}\right)\right)
$$

where $x_{i, j}^{t+1}$ and $x_{i, j}^{t}$ are the position of $j$ th dimension of particle $i$ in $t+1$ and $t$ iterations, respectively, and $\operatorname{randn}(0, \sigma)$ is a random number of Gaussian distribution whose variance is $\sigma^{2}$. The parameter $\sigma^{2}$ can be calculated [16].

$$
\sigma^{2}=\left\{\begin{array}{c}
1, \text { if } f_{i} \leq f_{j} \\
e^{\left(\frac{\left(f_{k}-f_{i}\right)}{\left|f_{i}\right|+\varepsilon}\right)}, \text { otherwise } k \in[1, N], k \neq i
\end{array}\right.
$$

Where $i, \mathrm{j} \in[1, r$ size $]$ and $i \neq \mathrm{j}$. $r$ size represents the number of rooster swarms. $f_{i}$ and $f_{j}$ are the fitness values of rooster $i$ and $\mathrm{j}$, respectively; $\xi$ represents a number which is few adequate [16].

Some hens can rob good food from another group

$$
\begin{array}{r}
x_{i, j}^{t+1}=x_{i, j}^{t}+S 1 \times \text { Rand } \times\left(x_{r 1, j}^{t}-x_{i, j}^{t}\right) \\
+S 2 \times \text { Rand } \times\left(x_{r 2, j}^{t}-x_{i, j}^{t}\right)(7)
\end{array}
$$

Where $x_{r 1, j}^{t}$ and $x_{r 2, j}^{t}$ are the position of rooster individual $r 1$ in the population of hen $x i$ and rooster individual $r 2$ in the other population, respectively. Rand is a uniform random number over $[0,1] . S 1$ and $S 2$ indicate the weight calculated [16].

$$
S 1=e^{\left(\frac{\left(f_{i}-f_{r 1}\right)}{\left|f_{i}\right|+\varepsilon}\right)}
$$

And

$$
S 2=e^{\left(f_{r 2}-f_{i}\right)}
$$

Where $f_{r} 1$ and $f_{r} 2$ are respectively, the fitness value of rooster individual $r 1$ in the population of hen $x i$ and rooster individual $r 2$ in the other population [16]. Chicks search for food beside their mothers

$$
x_{i, j}^{t+1}=x_{i, j}^{t}+F L \times\left(x_{m, j}^{t}-x_{i, j}^{t}\right)
$$

Where FL stands for a parameter, meaning that the chick would follow its mother to forage for food. $x_{m, j}^{t}$ represents the position of the $i$ th chick's mother ( $m$ $\in[1, N])[16]$.

\subsection{Genetic algorithm (GA)}

The Genetic Algorithm (GA) technique is a group of computational processes model stimulated by evolution. This algorithm uses a chromosome-like data construction to find a potential and optimal solution for a given specific problem. The GA was first defined and developed by J. Hollan, also the GA can be considered as an evolutionary algorithm appropriate for solving such scheduling problems [3, 17] and it is a very good optimization technique that emphasizes through several phase like selection, crossover and mutation to detect an optimal solution. Genetic Algorithm simulates the procedure of natural selection using bio-inspired operators such as crossover and mutation.

It depends on the theory of survival of the fittest. Genetic Algorithm considers a population of solutions (individuals). The fitness of each solution is determined by evaluating a fitness function against each solution. The survival of the individual to the next iteration is absolutely based on the fitness value of the individual. The individuals with least fitness value will be rejected from the population. Great feature gain from parent solutions are propagated to the next generation by applying crossover and mutation [7]. The pseudo code for evolutionary algorithm is shown in the Fig. 3.

Crossover is a genetic operator used to turn the chromosomes from one generation to the next. It is definition to reproduction and biological crossover, onto which genetic algorithms are based. Both implemented crossovers don't do mutual exchange of genetic material between two parents. The creation of new child depends on the information that was taken from one individual and inserts it in the other individual. Also in genetic algorithms, mutation is a genetic operator used to preserve genetic difference from one generation of a population of chromosomes to the next. It is identical to biological mutation. The probability which mutations will take place and if mutation takes place at all can be configured [3]. 


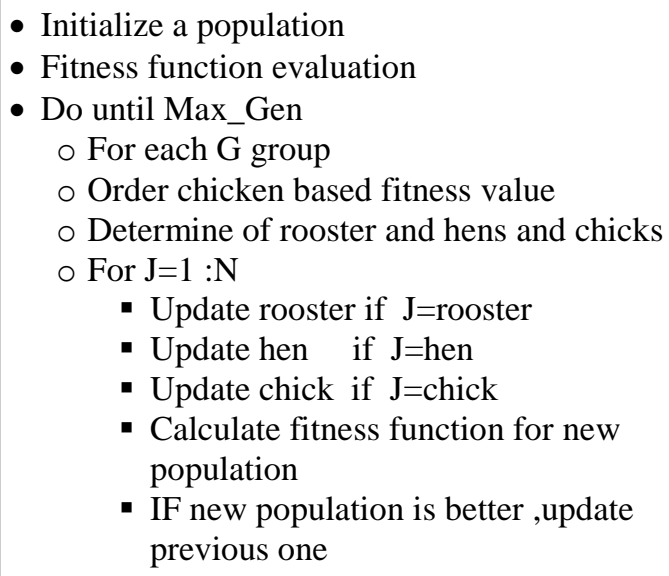
population

- IF new population is better, update previous one

Figure. 2 Chicken swarm optimization pseudo code

\subsection{Particle swarm optimization (PSO)}

Particle Swarm Optimization (PSO) is a global optimization technique proposed by Kennedy and Eberhart (1995). A swarm consist of a group of particles that each particle represents a possible solution.

Suppose that each solution can be considered as a point in N-Dimensional space that each point or particle has start velocity, particles move through solution space, and after each time step, particles are rated according to some fitness criterion. They are accelerated towards particle with best fitness value within their communication group. These movies of PSO help particles escape from local optimal solutions. Each particle has a simple memory that remember the position of best solution achieve by itself, this value is called personal best (pbest) and the position of best solution obtained so far by any particle in the neighbourhood of that particle, known as global best (gbest). The basic concept of PSO lies in accelerating each particle towards its pbest and the gbest locations, with a random weighted acceleration at each time step [18].The formulas for updating the velocity of each particle in the swarm are as follows.

$$
\begin{aligned}
V_{i d}(t+1)= & W V_{i d}(t)+c 1 r 1\left(P_{i d}-X_{i d}\right) \\
& +c 2 r 2\left(P_{g d}-X_{i d}\right)
\end{aligned}
$$

Where

$\mathbf{V}_{\text {id }}$ : velocity of dimension d of the $i$ th particle.

$\mathbf{P}_{\text {id }}$ : personal best previous position of the $i$ th Particle. $\mathbf{P}_{\mathrm{gd}}$ : the global best position for all particles.

$\mathbf{X}_{\text {id }}$ : current position of the $i$ th particle.

c1 \& c2 : are acceleration constants.

$\mathbf{r 1 ,} \mathbf{r 2}$ : random function in the range $[0,1]$.

W : Inertia weight.
1. $P \leftarrow$ Generate Initial Population ();

2. Evaluate $(P)$;

3. while !Stop Condition() do

4. $\quad P^{\prime} \leftarrow$ Select Parents $(P)$;

5. $P^{\prime} \leftarrow$ Apply Variation Operators $\left(P^{\prime}\right)$;

6. Evaluate $\left(P^{\prime}\right)$;

7. $P \leftarrow$ Select New Population $\left(P, P^{\prime}\right)$;

8. end while

9. Result: The best solution found

Figure. 3 The pseudo code for genetic algorithm

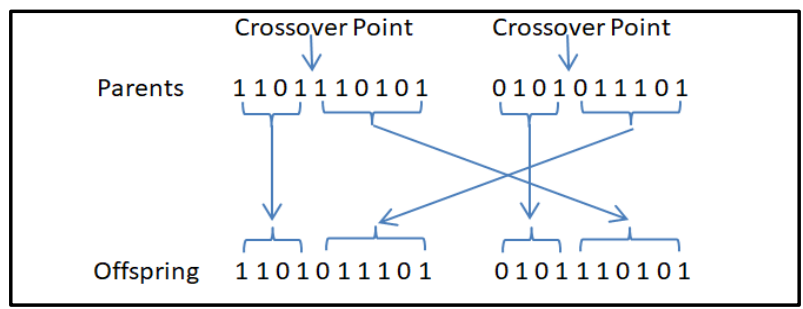

Figure. 4 Crossover example

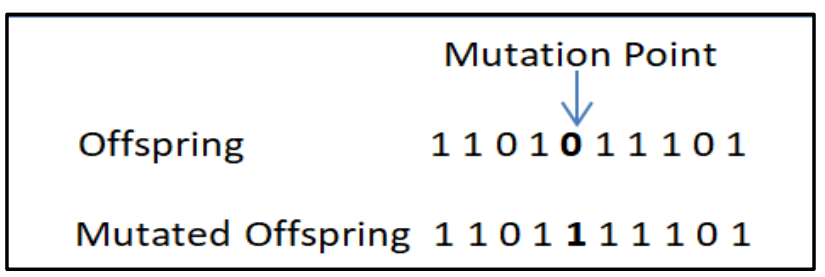

Figure. 5 Mutation example

The new velocity calculated in that iteration is used for updating the current position of each particle by using the following formula:

$$
\boldsymbol{X}_{i d}(t+1)=X_{i d}(t)+V_{i d}(t+1)
$$

Where

$\mathbf{X}_{\text {id }}(\mathrm{t})$ : current position of the $i$ th particle.

$\mathbf{X}_{\text {id }}(t+1)$ : new position of the $i$ th particle.

$\mathbf{V}_{\text {id }}(t+1)$ : New velocity of the $i$ th particle.

The process of updating the velocity and the position of each particle in the swarm is repeated until it reached a specific number of iterations as a termination criteria or reaching to the same final solution [18].

\section{Proposed work}

CVRP will be solved by using new hybrid Algorithm CSO, Tabu Search and Genetic Algorithm (Crossover and Mutation operations both or one of them) (HYCSOGATS) which will be listed in the next sections. 


\subsection{Hybrid CSO with GA and TS for CVRP}

The CVRP will be solved in this part by using CSO with GA and TS, and as we mentioned earlier, one of the advantages of using TS is that it helps to avoid local optima where all the neighbouring solutions are non-improving. This advantage will be used with roosters and dominated hens to get better results. Also we will use the advantages of GA in applying an iterative and stochastic process on a group of individuals (population), where each individual represents a potential solution to the problem [3]. To measure their aptitude for the problem, the individuals are assigned a fitness value. This value represents the quantitative information used by the algorithm to guide the search. The tradeoff between exploration of new areas of the search space and exploitation of good solutions accomplished by this kind of algorithms is one of the key factors for their high performance with respect to other meta-heuristics. This exploration/exploitation balance can be sharpened with some different parameters of the algorithm such as the population used (decentralized or not), the variation operators applied, or the probability of applying them, among others. In the first, initialize a population of chickens and define related parameters as flow (The number of iterations - the number of populations in the swarm the number of each roosters, hens, and chicks in the population - the coordinates of each customer - the capacity of vehicles - specify some constants like flow mother - the initial solutions- Crossover Percentage- Number of Offsprings- Mutation Percentage- Mutation Rate). After that we will generate a random solution and calculate the fitness for this solution, then we apply the Tabu Search and calculate the fitness, after that we choose the best fitness value. According to previous results we will update the hierarchical order for chickens. After initialization steps the next steps repeated until the number of iterations is finished. In this case we have some roosters, hens, and chicks. We will apply CSO moving equations on the current iteration. Then we apply the Crossover operation on hens only and the Mutation operation on chicks only according to the chosen operation to get $\mathrm{t}$ solution, which means if the chosen operation is the Crossover then the algorithm used will be (HYCSOCRTS) or if the chosen operation is the Mutation then the algorithm used will be (HYCSOMUTS) or if the chosen operation is both (Crossover \& Mutation) then the algorithm used will be (HYCSOGATS). Then we calculate the fitness value for $t$ solution and compare it with the fitness of the previous iteration solution and choose best solution which has best fitness value and so on. The algorithm is described as follows in Fig. 6.

\section{Experimental results}

In this section, we present the implementation of our proposed methodologies. Experimental results using different sets of parameters are shown along with explanations for the results values. A comparison between Particle Swarm Optimization (PSO), Chicken Swarm Optimization (CSO), Hybrid CSO with genetic operators (Crossover and Mutation) (HYCSOGATS), Hybrid CSO with Crossover (HYCSOCRTS) and Hybrid CSO with Mutation (HYCSOMUTS) results for solving CVRP also provided. The comparison is made upon the results of experiments applied on well-known benchmarks. Our methodology is implemented using the following technologies.

Software: Matlab R2013a, Windows 10 Pro 64-bit operating system, Microsoft Excel.

Hardware: Intel(R)Core(TM)i7-5500U@2.40GHz machine, 16 GB RAM, Intel(R)HD Graphics 5500, AMD Radeon(TM) R9 M375.

We will compare the proposed algorithms HYCSOGATS, HYCSOCRTS and HYCSOMUTS algorithms by using three different classes of NEO benchmark datasets Augerat (A, B, P) of CVRP with instances per class [8] also with CSO [9], PSO . The instance details are shown in the Table 1.

From Table 1 it appears that in class A; 5, 6, 6, 7, and 10 vehicles are assigned to $31,36,44,53$, and 79 customers respectively and there is one node in each instance for warehouse and the capacity for each vehicle is 100 . Moreover, in class B; 5, 5 and 9 vehicles are assigned to 34,38 , and 56 customers respectively and there is one node in each instance for depot and the capacity of each vehicle is 100 . Finally, in class $\mathrm{P} ; 8$, and 15 vehicles are assigned to 15 and 59 customers respectively and the capacity of first vehicle is 35 and for second one is 80 .

The population structure is the main difference between our proposed algorithms, so we list different parameters' values used for the proposed algorithms in Table 2. In the Table 3, we compare CSO against best known results in the swarm fields and we consider the cost factor as will be indicated also as shown in the Fig. 7 and Fig. 8. As the Table 3, Fig. 7, and Fig. 8 which explain that we solve CVRP by a new technique CSO algorithm which is solved completely and we observe that the PSO algorithm is better than CSO algorithm. We indicate to solve a minimization problem so, in our graphs less results is better for that, we tried to enhance our results of CSO by combining it with genetic operators (Crossover, 


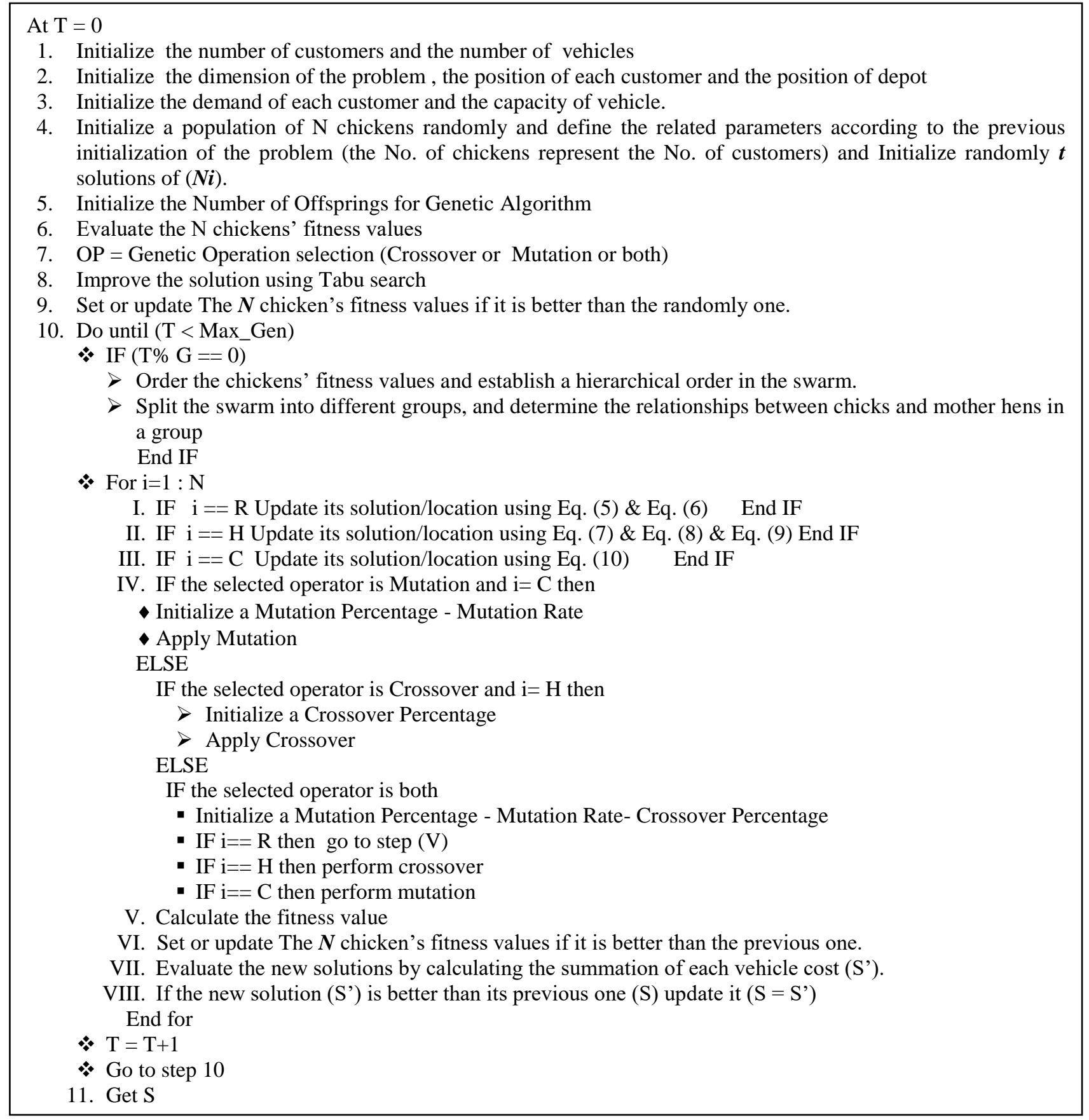

Figure. 6 HYCSOGATS for solving CVRP

Mutation) and TS as a hybrid algorithm which appears also in the Table 3. As the Table 3 and Fig. 7 and Fig. 8 we found that the costs of hybrid CSO with mutation, TS algorithm and hybrid CSO with Crossover, Mutation, TS algorithm are always better than PSO. We compare our obtained results against to the best know results in the literature as any accurate work should do. Also as shown in Table 3 and Fig. 8 the best cost of Hybrid CSO with Mutation, TS algorithm and Hybrid CSO with Crossover, Mutation, TS Algorithm is always better than benchmark cost [8] and it's overcome the benchmark results through 100 runs represented as Hit rate and our results has overcome the best results known (benchmark) completely. For CSO and hybrid CSO with Crossover, TS algorithm the costs also better than benchmark results except 2 instances $\mathrm{C} 7$ and C10. Also in Fig. 9 which contain a comparison between all algorithms in this paper by using relation between average cost for 100 run and number of iterations (1000 Iterations), we can observe that the HYCSOMUTS and HYCSOGATS is always better than the PSO. 


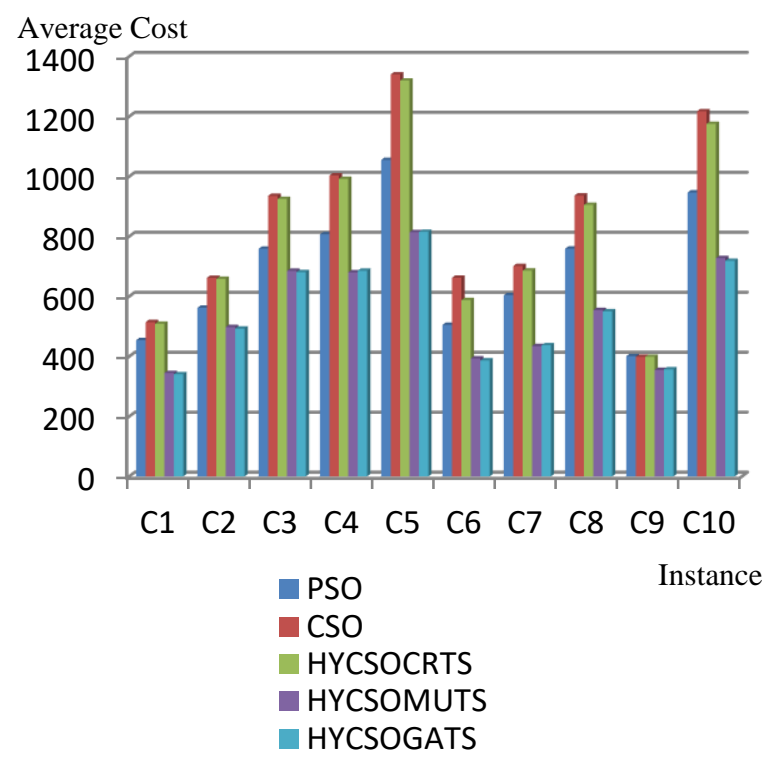

Figure. 7 Average costs for PSO, CSO, and hybrid CSO algorithms (less is better)

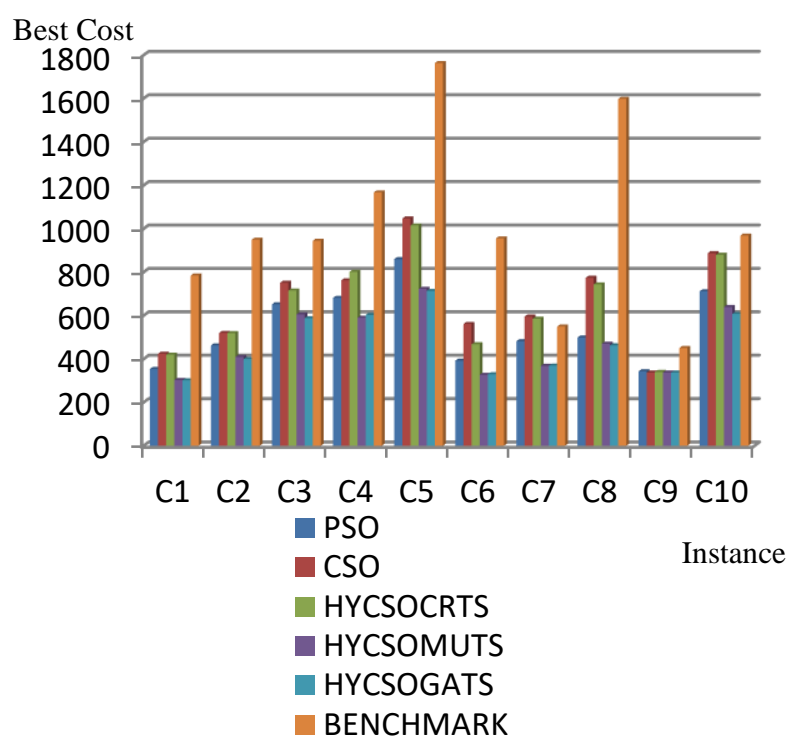

Figure. 8 Best costs for benchmark, PSO, CSO, and hybrid CSO algorithms (less is better)

Table 1. The instances details

\begin{tabular}{||c|c|c|c|c|c|c||}
\hline $\begin{array}{c}\text { Instance } \\
\text { Symbol }\end{array}$ & $\begin{array}{c}\text { Problem } \\
\text { Name }\end{array}$ & $\begin{array}{c}\text { No. of } \\
\text { Nodes }\end{array}$ & $\begin{array}{c}\text { No. of } \\
\text { Customers }\end{array}$ & $\begin{array}{c}\text { Vehicles } \\
\text { count }\end{array}$ & $\begin{array}{c}\text { Vehicle } \\
\text { capacity }\end{array}$ & Type \\
\hline C1 & A_N32_K5 & 32 & 31 & 5 & 100 & A \\
\hline C2 & A_N37_K6 & 37 & 36 & 6 & 100 & A \\
\hline C3 & A_N45_K6 & 45 & 44 & 6 & 100 & A \\
\hline C4 & A_N54_K7 & 54 & 53 & 7 & 100 & A \\
\hline C5 & A_N80_K10 & 80 & 79 & 10 & 100 & A \\
\hline C6 & B_N35_K5 & 35 & 34 & 5 & 100 & B \\
\hline C7 & B_N39_K5 & 39 & 38 & 5 & 100 & B \\
\hline C8 & B_N57_K9 & 57 & 56 & 9 & 100 & B \\
\hline C9 & P_N16_K8 & 16 & 15 & 8 & 35 & P \\
\hline C10 & P_N60_K15 & 60 & 59 & 15 & 80 & P \\
\hline \hline
\end{tabular}

Table 2. Parameters' values

\begin{tabular}{||l|l||}
\hline Parameter & Value \\
\hline Number of iterations & 1000 \\
\hline Population size & 100 \\
\hline Dimensions & From instances \\
\hline \multicolumn{2}{|c|}{ PSO } \\
\hline Inertia Weight & 1 \\
\hline Inertia Weight Damping Ratio & 0.99 \\
\hline Personal Learning Coefficient & 2 \\
\hline Global Learning Coefficient & 2 \\
\hline Maximum Velocity & 0.1 \\
\hline Minimum Velocity & -0.1 \\
\hline \multicolumn{2}{|c|}{ CSO } \\
\hline Roosters Percentage & $15 \%$ \\
\hline
\end{tabular}

\begin{tabular}{|l|l|}
\hline Hens Percentage & $70 \%$ \\
\hline Chicks percentage & $15 \%$ \\
\hline \multicolumn{2}{|c|}{ Genetic } \\
\hline Crossover Percentage & 0.7 \\
\hline $\begin{array}{l}\text { Extra Range Factor for } \\
\text { Crossover }\end{array}$ & 0.4 \\
\hline Mutation Percentage & 0.3 \\
\hline Mutation Rate & 0.01 \\
\hline
\end{tabular}

\section{Conclusion}

In this paper we provide a new solution to the problem of capitated vehicle routing problem (CVRP) by using a hybrid algorithm that integrate between CSO algorithm and the GA. CVRP is an 
Table 3. Comparison between PSO results and hybrid CSO

\begin{tabular}{|c|c|c|c|c|c|c|c|}
\hline $\begin{array}{l}\text { Instance } \\
\text { Symbol }\end{array}$ & Algorithm & Mean Cost & $\begin{array}{c}\text { Maximum } \\
\text { Cost }\end{array}$ & $\begin{array}{l}\text { Minimum } \\
\text { Cost }\end{array}$ & $\begin{array}{l}\text { Standard } \\
\text { Deviation }\end{array}$ & $\begin{array}{c}\text { Hit } \\
\text { rate } \%\end{array}$ & $\begin{array}{c}\text { Best Known } \\
\text { (Benchmark) }\end{array}$ \\
\hline \multirow{5}{*}{$\mathrm{C} 1$} & PSO & 454.307033 & 559.7693 & 353.534 & 46.00261 & 100 & \multirow{5}{*}{784} \\
\hline & $\mathrm{CSO}$ & 514.604359 & 594.6326 & 423.6803 & 37.65053 & 100 & \\
\hline & HYCSOCRTS & 509.1024 & 622.4166 & 419.3538 & 41.73381 & 100 & \\
\hline & HYCSOMUTS & 344.8993 & 432.1224 & 302.7995 & 26.23513 & 100 & \\
\hline & HYCSOGATS & 340.7837 & 400.5959 & 301.0805 & 19.46375 & 100 & \\
\hline \multirow{5}{*}{$\mathrm{C} 2$} & PSO & 562.4223 & 658.0035 & 461.8879 & 37.40148 & 100 & \multirow{5}{*}{949} \\
\hline & $\mathrm{CSO}$ & 662.0233 & 845.8991 & 519.5303 & 57.8781 & 100 & \\
\hline & HYCSOCRTS & 658.8917 & 847.1445 & 518.3605 & 55.32069 & 100 & \\
\hline & HYCSOMUTS & 498.089 & 632.4186 & 408.8499 & 36.44005 & 100 & \\
\hline & HYCSOGATS & 492.9874 & 607.6489 & 399.3542 & 45.33578 & 100 & \\
\hline \multirow{5}{*}{$\mathrm{C} 3$} & PSO & 759.4891 & 905.6364 & 651.3552 & 58.32224 & 100 & \multirow{5}{*}{944} \\
\hline & $\mathrm{CSO}$ & 935.632 & 1306.743 & 750.6594 & 92.86331 & 56 & \\
\hline & HYCSOCRTS & 925.3248 & 1236.854 & 714.7642 & 86.53806 & 62 & \\
\hline & HYCSOMUTS & 685.710515 & 766.3425 & 605.1577 & 34.20851 & 100 & \\
\hline & HYCSOGATS & 681.274559 & 799.9469 & 587.4775 & 41.48588 & 100 & \\
\hline \multirow{5}{*}{$\mathrm{C} 4$} & PSO & 807.6556 & 946.2357 & 681.1021 & 59.03508 & 100 & \multirow{5}{*}{1167} \\
\hline & $\mathrm{CSO}$ & 1003.5489 & 1314.438 & 761.9232 & 98.07118 & 94 & \\
\hline & HYCSOCRTS & 992.333163 & 1248.799 & 800.8694 & 88.44271 & 97 & \\
\hline & HYCSOMUTS & 680.6451 & 789.9855 & 589.5107 & 34.73992 & 100 & \\
\hline & HYCSOGATS & 685.8487 & 817.5731 & 602.0497 & 34.00751 & 100 & \\
\hline \multirow{5}{*}{$\mathrm{C} 5$} & PSO & 1055.457 & 1254.06 & 859.3825 & 83.94576 & 100 & \multirow{5}{*}{1763} \\
\hline & $\mathrm{CSO}$ & 1340.357 & 1826.731 & 1046.661 & 153.8499 & 98 & \\
\hline & HYCSOCRTS & 1320.156 & 1774.346 & 1014.775 & 136.1512 & 99 & \\
\hline & HYCSOMUTS & 814.58946 & 902.2708 & 722.6783 & 42.59461 & 100 & \\
\hline & HYCSOGATS & 815.543415 & 1005.318 & 712.7805 & 45.91423 & 100 & \\
\hline \multirow{5}{*}{ C6 } & PSO & 505.217 & 620.899 & 391.6075 & 37.74062 & 100 & \multirow{5}{*}{955} \\
\hline & $\mathrm{CSO}$ & 662.569199 & 758.0507 & 560.7559 & 42.98174 & 100 & \\
\hline & HYCSOCRTS & 587.88933 & 699.4382 & 467.1473 & 48.18598 & 100 & \\
\hline & HYCSOMUTS & 393.6137 & 467.3745 & 326.364 & 31.93803 & 100 & \\
\hline & HYCSOGATS & 386.6958 & 495.8733 & 328.8926 & 32.79038 & 100 & \\
\hline \multirow{5}{*}{$\mathrm{C} 7$} & PSO & 604.399591 & 705.7596 & 481.1474 & 46.84487 & 10 & \multirow{5}{*}{549} \\
\hline & $\mathrm{CSO}$ & 701.655 & 793.5782 & 594.9109 & 44.74526 & 0 & \\
\hline & HYCSOCRTS & 686.3594 & 789.4614 & 584.7762 & 44.70158 & 0 & \\
\hline & HYCSOMUTS & 434.608005 & 521.3993 & 367.8033 & 36.78653 & 100 & \\
\hline & HYCSOGATS & 437.528519 & 553.2995 & 368.995 & 35.39143 & 99 & \\
\hline \multirow{5}{*}{$\mathrm{C} 8$} & PSO & 759.5568 & 950.7142 & 498.2958 & 61.82116 & 100 & \multirow{5}{*}{1598} \\
\hline & $\mathrm{CSO}$ & 936.621093 & 1179.3586 & 773.5207 & 77.49575 & 100 & \\
\hline & HYCSOCRTS & 905.559 & 1113.983 & 743.1081 & 67.605 & 100 & \\
\hline & HYCSOMUTS & 554.781161 & 652.1262 & 469.644 & 42.96788 & 100 & \\
\hline & HYCSOGATS & 550.759535 & 644.9764 & 462.3205 & 40.63339 & 100 & \\
\hline \multirow{5}{*}{ C9 } & PSO & 401.074 & 555.9745 & 342.4674 & 40.65797 & 89 & \multirow{5}{*}{450} \\
\hline & $\mathrm{CSO}$ & 398.96339 & 483.3567 & 336.925 & 37.76741 & 88 & \\
\hline & HYCSOCRTS & 399.026387 & 497.7501 & 339.6567 & 36.49407 & 88 & \\
\hline & HYCSOMUTS & 355.08111 & 426.5276 & 336.925 & 16.30825 & 100 & \\
\hline & HYCSOGATS & 357.222 & 439.1826 & 336.925 & 20.99526 & 100 & \\
\hline \multirow{5}{*}{$\mathrm{C} 10$} & PSO & 947.0628 & 1408.375 & 711.2227 & 110.496 & 59 & \multirow{5}{*}{968} \\
\hline & $\mathrm{CSO}$ & 1217.715 & 1613.475 & 886.7962 & 165.5583 & 5 & \\
\hline & HYCSOCRTS & 1175.446 & 1494.49 & 879.4456 & 143.83 & 9 & \\
\hline & HYCSOMUTS & 727.8232 & 845.5919 & 638.7676 & 46.1496 & 100 & \\
\hline & HYCSOGATS & 718.892588 & 811.3717 & 606.8586 & 43.02001 & 100 & \\
\hline
\end{tabular}




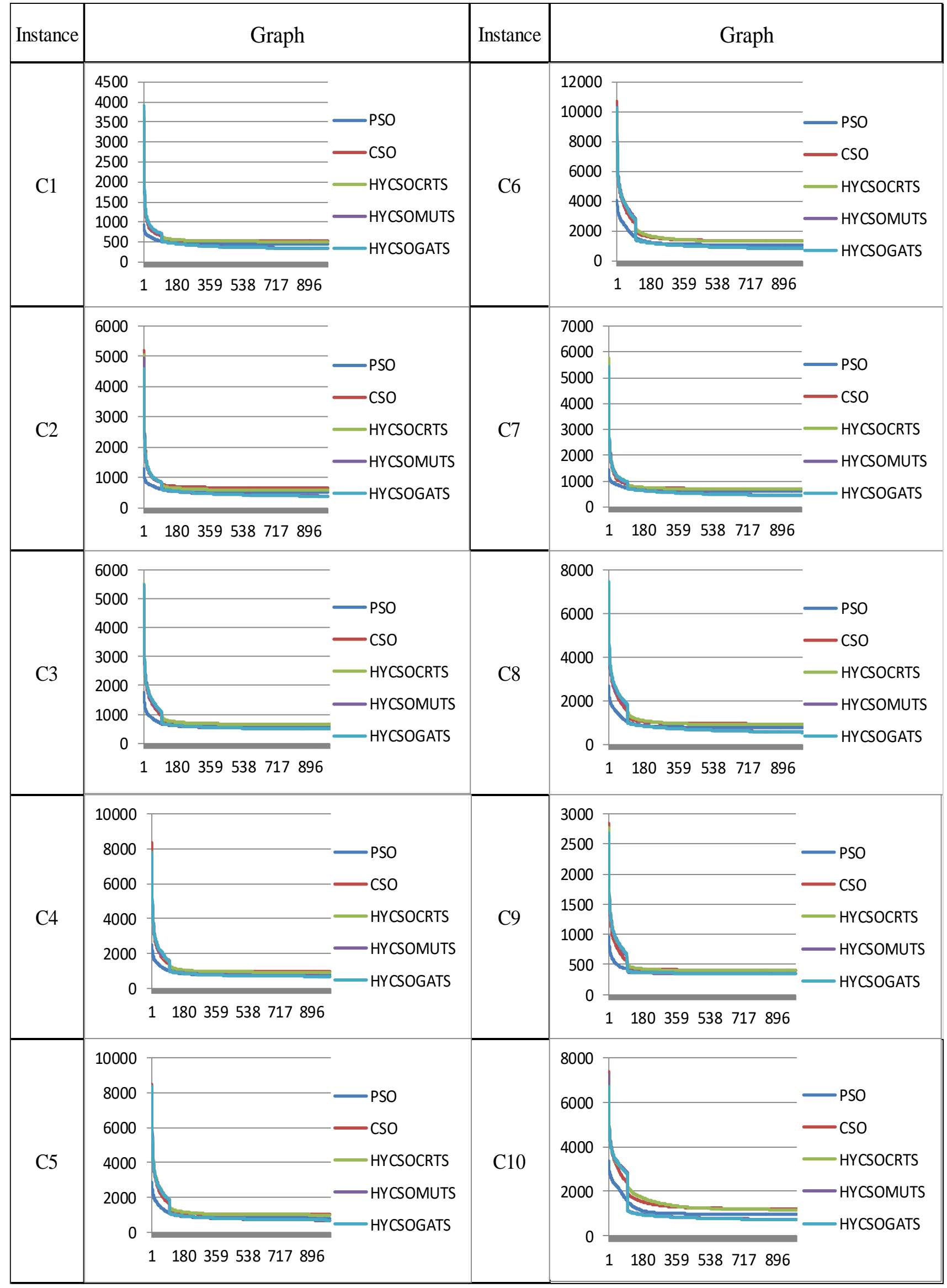

Figure. 9 Between algorithms by using relation between average cost and number of iteration 
integer programming problem, which is fall into the category of NP- hard problem with the goal of minimizing the total travelled distance by the involved capacity - limited vehicles to reach their destination and back to the start depot. We also compared the results CSO against PSO in solving CVRP, from the results we found that the PSO algorithm is better in cost than CSO algorithm. So we improved our solution by create a new hybrid algorithm by combining CSO with TS and GA using a Crossover and Mutation operators (HYCSOGATS). Then we found the best cost of (HYCSOGATS) is always better than benchmark and PSO costs by $100 \%$.

\section{Conflicts of Interest}

The authors declare no conflict of interest.

\section{Author Contributions}

Conceptualization, Nora, Ahmed, and Mahmoud; methodology, Ahmed; software, Nora; validation, Nora, Ahmed, and Mahmoud; formal analysis, Ahmed, and Mahmoud; investigation, Nora; resources, Nora, and Ahmed; data curation, Nora; writing - original draft preparation, Nora, and Ahmed; writing - review and editing, Nora, and Mahmoud; visualization, Nora, and Ahmed; supervision, Mahmoud.

\section{References}

[1] R. Linfati and J. Escobar, "Reoptimization Heuristic for the Capacitated Vehicle Routing Problem", Journal of Advanced Transportation, Vol. 2018, No. 3743710, pp. 1-8, 2018.

[2] S. Kumar and R. Panneerselvam, "A Survey on the Vehicle Routing Problem and Its Variants", Intelligent Information Management, Vol. 04, No. 03, pp. 66-74, 2012.

[3] E. Alba and B. Dorronsoro, Cellular Genetic Algorithms, Vol.42, Springer, 2008.

[4] X. Meng, Y. Liu, X. Gao, and H. Zhang, "A New Bio-inspired Algorithm: Chicken Swarm Optimization", In: Proc. International Conf. in Swarm Intelligence, Hefei, China , pp. 86-94, 2014.

[5] F. Glover and G. A. Kochenberger, Handbook of Metaheuristics, Vol. 57, Springer, 2003.

[6] L. Calvet, D. Wang, A. Juan, and L. Bové, "Solving the multidepot vehicle routing problem with limited depot capacity and stochastic demands", Int. Trans. Oper. Res., Vol. 26, No. 2, pp. 458-484, 2019.
[7] G. Nagy and S. Salhi, "Heuristic algorithms for single and multiple depot vehicle routing problems with pickups and deliveries", European Journal of Operational Research, Vol. 162, No. 1, pp.128, 2005.

[8] http://neo.lcc.uma.es/vrp/vehicle-routingproblem/, 07-February-2020.

[9] N. Niazy, A. El-sawy, and M. Gadallah, "A Hybrid Chicken Swarm Optimization with Tabu Search Algorithm for Solving Capacitated Vehicle Routing Problem", International Journal of Intelligent Engineering and Systems, Vol. 13, No. 4, pp. 237-247, 2020.

[10] W. Geng, "Cognitive Deep Neural Networks prediction method for software fault tendency module based on Bound Particle Swarm Optimization”, Cognitive Systems Research, Vol. 52, No. 2018, pp. 12-20, 2018.

[11] Y. Kao and M. Chen, "A hybrid PSO algorithm for the CVRP problem", In: Proc. International Conf. in Evolutionary Computation Theory and Applications, pp. 539-543, 2011.

[12] K. Bhattacharjee and M. Pant, "Hybrid particle swarm optimization-genetic algorithm trained multi-layer perceptron for classification of human glioma from molecular brain neoplasia data”, Cognitive Systems Research, Vol. 58, No. 2019, pp. 173-194, 2019.

[13] J. Li, Y. Xia, B. Li, and Z. Zeng, "A pseudodynamic search ant colony optimization algorithm with improved negative feedback mechanism", Cognitive Systems Research, Vol. 62, No. 2020, pp. 1-9, 2020.

[14] Y. Zhang, M. Qi, L. Miao, and G. Wu, "A generalized multi-depot vehicle routing problem with replenishment based on LocalSolver", International Journal of Industrial Engineering Computations, Vol. 6, No.1, pp. 81-98, 2015.

[15] F. Chebihi, M. E. Riffi, A. Agharghor, S. C. B. Semlali, and A. Haily, "Improved Chicken Swarm Optimization Algorithm to Solve the Travelling Salesman Problem", Indonesian Journal of Electrical Engineering and Computer Science, Vol. 12, No. 3, pp. 1054-1062, 2018.

[16] C. Qu, S. Zhao, Y. Fu, and W. He, "Chicken Swarm Optimization Based on Elite OppositionBased Learning", Mathematical Problems in Engineering Systems, Vol. 2017, No. 6, pp. 2, 2017.

[17] A. Hussein, N. ArunKumar, G. RamirezGonzalez, E. Abdulhay, J. Tavares, and V. Albuquerque, "A medical records managing and securing blockchain based system supported by a Genetic Algorithm and Discrete Wavelet 
Received: July 3, 2020. Revised: July 29, 2020.

Transform", Cognitive Systems Research, Vol. 52, No. 2018, pp. 1-11, 2018.

[18]M. Tavakoli and A. Sami, "Particle Swarm Optimization in Solving Capacitated Vehicle Routing Problem", Bull. Electr. Eng. Informatics, Vol. 2, No. 4, 2013. 